\title{
A test of SDSS aperture corrections using integral-field spectroscopy
}

\author{
Andrew W. Green, ${ }^{1 \star}$ Karl Glazebrook, ${ }^{2}$ David G. Gilbank, ${ }^{3}$ Peter J. McGregor, ${ }^{4} \dagger$ \\ Ivana Damjanov, ${ }^{5}$ Roberto G. Abraham ${ }^{6}$ and Rob Sharp ${ }^{4}$ \\ ${ }^{1}$ Australian Astronomical Observatory, PO Box 915, North Ryde, NSW 1670, Australia \\ ${ }^{2}$ Centre for Astrophysics and Supercomputing, Swinburne University, Hawthorn, VIC 3122, Australia \\ ${ }^{3}$ South African Astronomical Observatory, PO Box 9, Observatory 7935, South Africa \\ ${ }^{4}$ Research School of Astronomy and Astrophysics, Australian National University, Cotter Rd, Weston, ACT 2611, Australia \\ ${ }^{5}$ Harvard-Smithsonian CfA, 60 Garden St, MS-20, Cambridge, MA 02138, USA \\ ${ }^{6}$ Department of Astronomy and Astrophysics, University of Toronto, 50 St George St, Toronto, ON M5S 3H4, Canada
}

Accepted 2017 May 8. Received 2017 May 8; in original form 2015 August 20

\begin{abstract}
Corrections for fibre aperture losses in modern surveys such as the Sloan Digital Sky Survey are at the foundation of statistical studies of galaxy properties, yet these corrections are not well understood. We compare direct measurements of the total (aperture-free) $\mathrm{H} \alpha$-based star formation rate from integral-field spectroscopy with Brinchmann et al. and Gilbank et al. derived estimates of the star formation rate from fibre-aperture spectroscopy for the same $z \simeq$ 0.07 star-forming galaxies drawn from the Sloan Digital Sky Survey. This comparison reveals that aperture-corrected star formation rates are underestimated for more highly star-forming galaxies: specifically by 0.3 and $0.6 \mathrm{dex}$ at rates of star formation of 10 and $100 \mathrm{M}_{\odot} \mathrm{yr}^{-1}$, respectively, while the underestimate vanishes at $1 \mathrm{M}_{\odot} \mathrm{yr}^{-1}$. Furthermore, previous estimates of the aperture loss for $\mathrm{H} \alpha$ emission only marginally correlate with direct measurements of the aperture loss for individual galaxies. The primary limitation of our work is the lack of spatially resolved dust attenuation corrections. We conclude that corrections for aperture losses should be considered with caution.
\end{abstract}

Key words: methods: observational-galaxies: fundamental parameters - galaxies: statistics.

\section{INTRODUCTION}

Modern statistical astronomy is almost universally driven by fibrebased multi-object spectroscopic surveys. For such surveys of galaxies with redshifts, $z$, less than 0.3 , a fibre rarely covers the whole extent of the optical emission of a galaxy, often missing two-thirds of the light of a galaxy at $z \simeq 0.1$ (e.g. Brinchmann et al. 2004). Recent key statistical properties of modern galaxies, such as the global star formation rate (SFR) density, mass-metallicity relation and mass-SFR relation, depend on understanding and, if necessary, correcting for this missing information in cases where the gradient is strong with galaxy radius.

In some cases, it is possible to confirm the statistical properties of galaxies using other methods. Estimates of the global SFR density of the Universe can be made using radio or ultraviolet surveys that are free from the limitations of a fibre aperture. However, not only are alternative methods not always available, but also the physics behind measurements at different wavelengths is often different.

\footnotetext{
${ }^{\star}$ E-mail: andrew.green@aao.gov.au
}

$\dagger$ Deceased.
Radio and ultraviolet surveys can not only measure the global SFR density, but also measure SFRs on different time-scales and rely on different physics than optical surveys. Therefore, it is of great interest to better understand the accuracy and systematics of aperture corrections that account for light not captured in fibre-based surveys.

There has already been considerable work to better understand how aperture losses may affect SFRs and how to correct for such an effect. Hopkins et al. (2003) characterized the aperture corrections needed for SFRs in the Sloan Digital Sky Survey (SDSS; York et al. 2000) using complementary estimates from $1.4 \mathrm{GHz}$ luminosities. Brinchmann et al. (2004) developed a comprehensive method for correcting SFRs using colour information for regions the fibre aperture cannot reach. Using data from the Nearby Field Galaxy Survey, which includes both aperture and integrated spectra, Kewley, Jansen \& Geller (2005) tested an aperture correction that is approximately the ratio of the fibre-to-total $r$-band flux. Salim et al. (2007) compare ultraviolet SFRs with those of Brinchmann et al. (2004) and find that the aperture-corrected rates of the latter agree well. More recently, Gilbank et al. (2010) comprehensively reviewed methods of estimating SFR and methods of aperture correction to enable more direct comparisons between low- and highredshift studies. SDSS remains the benchmark spectroscopic survey 
of nearby galaxies, but only integral-field spectroscopic surveys can test the aperture-loss corrections used.

Integral-field spectroscopy (IFS) offers the possibility for aperture-free or nearly aperture-free optical spectroscopy of nearby galaxies in large numbers. Gerssen, Wilman \& Christensen (2012) used IFS to directly measure the aperture correction for a sample of $\sim 100$ galaxies with equivalent width of $\mathrm{H} \alpha$ emission larger than $20 \AA$ and compare the correction with that estimated by SDSS. They show that Brinchmann et al. (2004) underestimated the aperture correction for SFR by a factor of $2.5(0.4 \mathrm{dex})$ for almost all galaxies in their sample. Intermediate-scale integral-field spectroscopic surveys in progress (SAMI Galaxy Survey, Bryant et al. 2015; Mapping Nearby Galaxies at APO (MaNGa), Bundy et al. 2015) will provide aperture-free data for many thousands of galaxies. However, integral-field spectroscopic surveys of as many galaxies as SDSS are still some years away (e.g. using the proposed Hector instrument on the Anglo-Australian Telescope), so more detailed studies of aperture effects using aperture-free data are needed to inform studies of samples where aperture effects are still a major concern.

In this paper, we review the accuracy of aperture corrections for SFRs in SDSS. The DYNAMO integral-field spectroscopic survey (Green et al. 2014) of $z<0.2$ SDSS galaxies provides nearly aperture-free spectroscopic information around the $\mathrm{H} \alpha$ emission line. A direct (aperture-free) measurement of the total SFR and the corresponding aperture correction from the SDSS fibre aperture can be made for each galaxy using this data set. We use such measurements to characterize the systematics of other aperture-correction techniques and assess the impact of such systematics on the measurement of the local SFR density.

Complementary to and contemporary with this paper is the work of Richards et al. (2016a), which is based on IFS from the SAMI Galaxy Survey. That work samples typically lower SFR galaxies than we consider here. It focuses more on reproducing and directly testing the aperture-loss corrections for SFRs of Brinchmann et al. (2004) and Hopkins et al. (2003) using Sydney-AAO Multi-object Integral-field spectrograph (SAMI) data and less on comparisons of the final SFR estimates derived by the various methods, as discussed here. The work of Richards et al. (2016a) is very complementary to this paper, and therefore we combine the two samples in Section 3.5.

The plan of the paper is as follows. Section 2 reviews several methods for determining SFRs. Section 3 starts by providing a framework for understanding aperture-loss corrections and reviewing several methods that have been popularly used. It then compares these methods with the direct measurement provided by the DYNAMO data. Section 3 concludes with how $\mathrm{H} \alpha$-based SFRs are affected by these corrections and also the impact on the local density of star formation. Section 4 presents our major findings and some brief discussion.

This paper uses the same initial mass function (IMF) and cosmology as Green et al. (2014), namely, the cosmology given by $H_{0}=71 \mathrm{~km} \mathrm{~s}^{-1} \mathrm{Mpc}^{-1}, \Omega_{\mathrm{M}}=0.27$ and $\Omega_{\lambda}=0.73$ and the Chabrier (2003) IMF.

\section{DATA}

For our analysis, we wish to compare SFRs of galaxies measured using IFS with those rates measured using fibre-aperture spectroscopy. The DYNAMO Survey (Green et al. 2014) provides both IFS and, because it is based on SDSS, fibre-aperture spectroscopy. From these data, we directly measure the aperture correction and

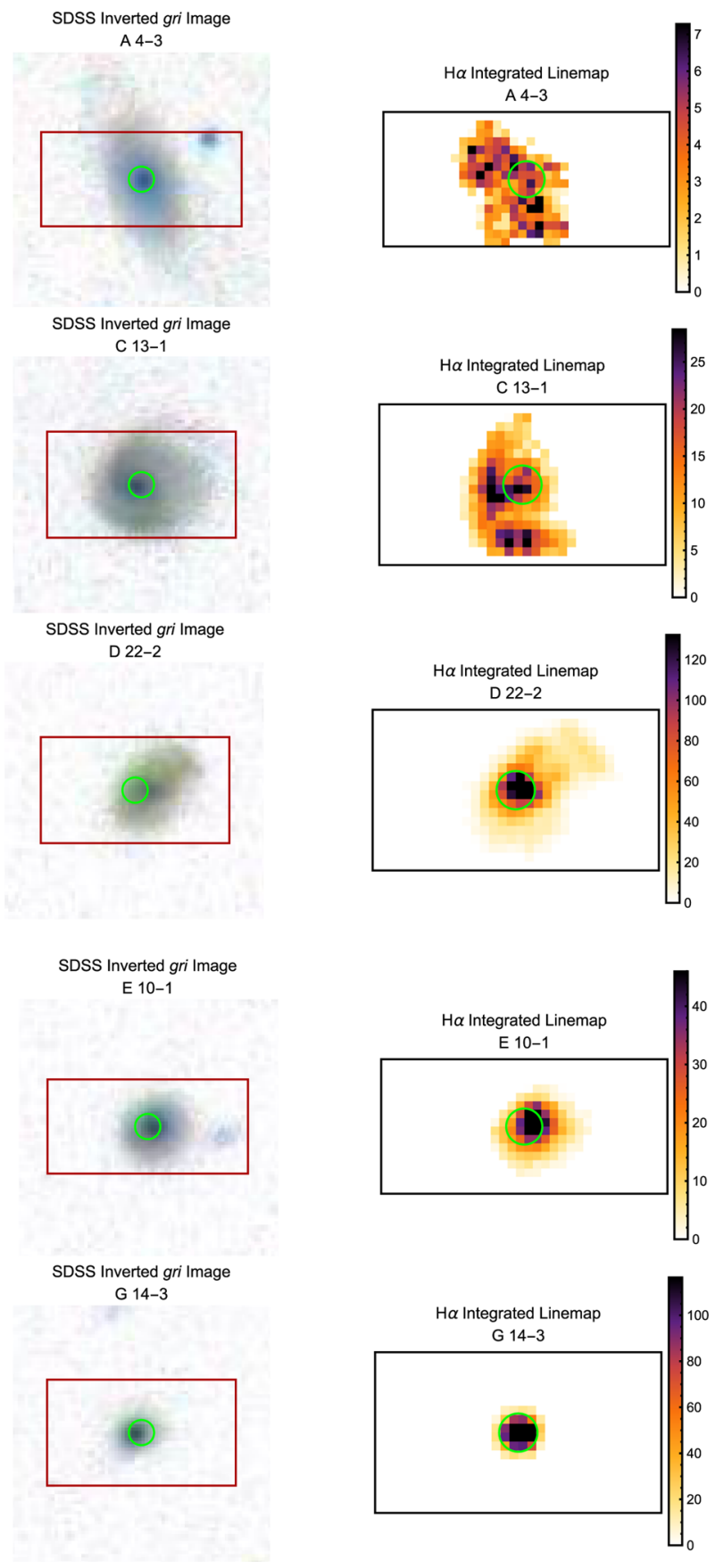

Figure 1. Examples of the field of view of the DYNAMO IFS and the SDSS fibre. The left-hand column shows the SDSS gri images (inverted) with the field of view of the IFS overlaid in red and the SDSS fibre overlaid in green. The right-hand column shows the $\mathrm{H} \alpha$ line map within the field of view of the IFS, again with the SDSS fibre aperture overlaid in green. The first two galaxies shown are chosen to exhibit some of the worst potential flux loss due to the limited field of view of the DYNAMO data. Others chosen to demonstrate more typical examples. Also apparent in many of these examples is the very limited coverage of the SDSS fibre. A similar presentation of all galaxies in our sample is included in the supplementary information of Green et al. (2014).

compare with the estimated corrections presented by Brinchmann et al. (2004) and by Gilbank et al. (2010). Fig. 1 shows examples of five galaxies used in our analysis and the fields of view of the DYNAMO IFS and the SDSS fibre-aperture spectroscopy. 


\subsection{DYNAMO star formation rates}

DYNAMO surveys a sample of star-forming galaxies drawn from SDSS and observed with IFS (Green et al. 2014). Data were collected using the SPIRAL+AAOmega spectrograph on the AngloAustralian Telescope, and the Wide-Field Spectrograph (WiFeS) spectrograph on the Australian National University 2.3-m telescope. The spectral resolution for WiFeS data was $R \sim 7000$, and $R \sim 10000$ for SPIRAL. Active galactic nuclei (AGN) have been excluded from the sample using techniques similar to Brinchmann et al. (2004). The sample can be divided roughly into two parts. The first part is a volume-limited sample of typical star-forming galaxies in the narrow redshift slice $0.055<z<0.084$. The second part is a sample of galaxies with extreme SFRs in a larger redshift range $z>0.124$. The galaxies in the combined sample cover a broad range in $\mathrm{H} \alpha$ luminosities $\left(10^{40}-10^{42} \mathrm{erg} \mathrm{s}^{-1}\right.$ within the SDSS fibre aperture). The sampling approach ensures there is roughly a uniform distribution of galaxies across the range of $\mathrm{H} \alpha$ luminosities or equivalently SFRs.

Included in the analysis here are all 67 galaxies of Green et al. (2014). In the figures of this paper, the galaxies from the volume limited, $0.055<z<0.084$ subset are shown with solid symbols, and the more extreme, higher redshift galaxies with open symbols.

The $\mathrm{H} \alpha$ fluxes from our IFS within the region covered by the SDSS fibre aperture are either fixed to, or agree with, fluxes from SDSS spectra. The $\mathrm{H} \alpha$ flux calibration of data from the SPIRAL instrument has been fixed to that of SDSS for the region of the galaxy covered by the fibre because of transparency and throughput stability issues with the SPIRAL data. Data from the WiFeS instrument do not have these issues, and instead are independently calibrated using spectrophotometric flux standards. WiFeS $\mathrm{H} \alpha$ fluxes within the SDSS fibre aperture and the SDSS spectrophotometry agree to a few per cent.

A few of the galaxies in the DYNAMO sample exhibit evidence for $\mathrm{H} \alpha$ emission beyond the field of view of the instrument. 22 of the 67 galaxies show some detectable flux in the 2-pixel border adjacent to the edge of the observed field of view. Of those, only two exhibit more than 10 per cent of the total observed flux in this border: A 8-4 with 12.2 per cent and A 4-3 (as shown in Fig. 1) with 11.0 per cent. 61 of the 67 galaxies show less than 4 per cent of the total flux in this 2-pixel border. Therefore, we are confident that 90 per cent of our sample excludes less than 10 per cent of the detectable $\mathrm{H} \alpha$ flux due to the limits of the field of view, and that two-thirds include all of the detectable flux.

Total SFRs for the DYNAMO galaxies are determined using the Kennicutt (1998) star formation law with a dust correction (see section 5.2 of Green et al. 2014 for exact details). The IFS covers at least $11 \times 22 \operatorname{arcsec}^{2}$ on the sky, larger than most of the galaxies included in our sample. Total $\mathrm{H} \alpha$ luminosities are determined by integrating the flux around the wavelength position of the $\mathrm{H} \alpha$ emission line, and spatially where the line is detectable (typically a flux limit of $\sim 1 \times 10^{-17} \mathrm{erg} \mathrm{s}^{-1} \mathrm{~cm}^{-2}$ ). The area of this region is comparable to the area of an aperture with the SDSS Petrosian radius measured in $r$ band. The luminosity is corrected for dust attenuation using the Balmer decrement method (ratio of $\mathrm{H} \alpha$ to $\mathrm{H} \beta$ ). The line ratio is taken from catalogue fluxes only within the SDSS fibre aperture as the IFS data are of insufficient quality around $\mathrm{H} \beta$. Our dust-correction method is therefore limited because it does not account for the possibility of the dust attenuation changing in the outer region relative to the central region, an effect that can (at least in principle) be accounted for in the methods described below. These dust-corrected luminosities are then scaled to SFRs with the
Kennicutt (1998) scale factor $\eta_{\mathrm{H} \alpha} \equiv L_{\mathrm{H} \alpha} / \mathrm{SFR}$. The SFRs for the DYNAMO sample are shown in the $\mathrm{SFR}_{\mathrm{IFS}}$ column of Table A1.

\subsection{SDSS star formation rates}

The SDSS includes fibre-based optical spectroscopy of nearby galaxies (median redshift $z \simeq 0.1$ ). The SDSS fibre covers the central 3-arcsec-diameter region of each galaxy. The luminosity of $\mathrm{H} \alpha$ emission in these spectra, and corresponding SFRs within the fibre have been measured for Data Release 4 (DR4) in the Value Added Catalog of Tremonti et al. (2004) and Brinchmann et al. (2004). DR4 was chosen because it was used in the selection of the initial sample. ${ }^{1}$ To determine the SFRs for purely star-forming galaxies included here, Brinchmann et al. use the Charlot et al. (2002) methodology. In this methodology, emission-line fluxes for each galaxy are fit to a model grid to determine simultaneously both the ratio of $\mathrm{H} \alpha$ luminosity to SFR, $\eta_{\mathrm{H} \alpha}$, the dust attenuation at $\mathrm{H} \alpha$, $A_{\mathrm{H} \alpha}$, and the metallicity of the gas. These SFRs are shown in the $\mathrm{SFR}_{\mathrm{B} 04}$ column of Table A1.

Brinchmann et al. (2004) compare their estimates of SFR with the fixed $\eta_{\mathrm{H} \alpha}$, Balmer decrement method employed for DYNAMO galaxies. Their $A_{\mathrm{H} \alpha}$ and $\eta_{\mathrm{H} \alpha}$ are similar to the fixed values commonly adopted (e.g. Kennicutt 1998). However, the values of $A_{\mathrm{H} \alpha}$ and $\eta_{\mathrm{H} \alpha}$ are seen to vary systematically with stellar mass, leading to some variation in estimated SFR with stellar mass as shown in their fig. 8, 'Method 4' (see also the discussion around fig. 4 of Gilbank et al. 2010). Their method predicts systematically more star formation (within the fibre aperture) than our method by $\approx 0.16 \mathrm{dex}$ across our sample.

The test of aperture corrections is simplified by removing any systematic differences in star formation rate estimates before comparing them. We convert Brinchmann et al. (2004) rates to the DYNAMO method because it is more commonly used. For each galaxy, we divide the B04 SFR by the 'Method 4' ratio given in their fig. 8 for the mass of the galaxy. The size of this correction in logarithmic units is given in the $\mathrm{SFR}_{\mathrm{B} 04}$-Correction column of Table A1. After removing this systematic, SFRs within the fibre for DYNAMO and Brinchmann et al. differ by only 0.05 dex on average, with scatter of 0.14 dex. An alternative approach would be to take the ratio of the fibre SFRs determined using the two methods for each galaxy. However, this ratio does not map straightforwardly on to the Brinchmann et al. aperture correction.

In addition to the SFR estimates of Brinchmann et al. (2004), Gilbank et al. (2010) also estimate aperture-corrected SFRs for SDSS galaxies in the 'Stripe 82' region using a variety of methods. For our analysis, we consider their ' $\mathrm{H} \alpha \lambda 6563$ SFR' (which is their preferred method), as it is most similar to that used for the DYNAMO estimates of SFR. The differences are: a slightly different IMF (described below); an intrinsic $\mathrm{H} \alpha$ to $\mathrm{H} \beta$ line ratio of 2.85 instead of $2.90^{2}$ and a different dust-extinction law. These differences result in only small differences in the final SFRs.

We have used a Chabrier (2003) IMF, and when comparing our SFRs with those of Brinchmann et al. (2004) and Gilbank et al. (2010), we scale their rates by 1.14 to account for their choice of a Kroupa (2001) IMF. The data used are summarised in Table 1.

\footnotetext{
${ }^{1}$ See Appendix A for a discussion of the changes introduced in the Data Release 7 (DR7) and their impact on our results.

22.90 is the value used by Calzetti, Kinney \& Storchi-Bergmann (1996).
} 
Table 1. Table of data used.

\begin{tabular}{|c|c|c|c|c|c|c|c|}
\hline \multirow[t]{2}{*}{ Galaxy ID } & \multirow[t]{2}{*}{$\begin{array}{c}\text { Stellar mass } \\
\left(\log \mathrm{M}_{\odot}\right)\end{array}$} & \multicolumn{3}{|c|}{$\begin{array}{c}\mathrm{SFR}_{\mathrm{B} 04} \\
\left(\log \mathrm{M}_{\odot} \mathrm{yr}^{-1}\right)\end{array}$} & \multicolumn{2}{|c|}{$\begin{array}{c}\mathrm{SFR}_{\mathrm{IFS}} \\
\left(\log \mathrm{M}_{\odot} \mathrm{yr}^{-1}\right)\end{array}$} & \multirow[t]{2}{*}{$\gamma(u$ band $)$} \\
\hline & & Total & Fibre & Correction & Total & Fibre & \\
\hline A $4-3$ & 10.63 & 0.25 & -0.25 & 0.34 & 0.22 & -0.68 & 4.28 \\
\hline A $4-4$ & 9.04 & -0.53 & -1.54 & 0.07 & -0.81 & -1.47 & 14.18 \\
\hline A $8-3$ & 9.80 & -0.09 & -0.86 & 0.12 & -0.14 & -1.00 & 10.21 \\
\hline A $8-4$ & 10.69 & 0.56 & -0.65 & 0.36 & -0.02 & -0.87 & 11.02 \\
\hline A $10-1$ & 10.58 & 0.17 & -0.02 & 0.32 & 0.23 & -0.31 & 6.70 \\
\hline A $10-2$ & 10.10 & -0.23 & -0.66 & 0.14 & -0.32 & -0.94 & 2.71 \\
\hline A $13-2$ & 9.64 & -0.36 & -1.16 & 0.12 & -0.79 & -1.35 & 9.25 \\
\hline B 4-4 & 10.06 & 0.30 & -0.60 & 0.14 & 0.48 & -0.72 & 3.84 \\
\hline B 4-3 & 10.50 & 0.59 & 0.06 & 0.29 & 0.62 & -0.46 & 4.69 \\
\hline B 8-4 & 9.67 & -0.17 & -0.84 & 0.12 & 0.42 & -0.91 & 6.00 \\
\hline B $8-3$ & 10.21 & -0.51 & -0.51 & 0.18 & 0.99 & -0.57 & 8.95 \\
\hline B $10-1$ & 9.59 & -0.07 & -0.92 & 0.12 & -0.26 & -0.90 & 4.78 \\
\hline B $11-2$ & 9.94 & 0.48 & -0.66 & 0.13 & 0.56 & -1.01 & 4.46 \\
\hline B $14-1$ & 10.35 & 0.47 & -0.34 & 0.22 & -0.17 & -0.55 & 7.90 \\
\hline B $15-1$ & 9.82 & 0.05 & -1.09 & 0.12 & -0.39 & -1.07 & 10.18 \\
\hline B $15-2$ & 9.86 & -0.14 & -0.65 & 0.12 & -0.30 & -0.78 & 7.23 \\
\hline B $20-1$ & 9.88 & 0.00 & -0.85 & 0.12 & -0.04 & -0.68 & 6.90 \\
\hline $\mathrm{C} 0-1$ & 9.11 & -0.41 & -0.70 & 0.09 & -0.39 & -0.74 & 3.62 \\
\hline C 4-2 & 9.49 & 0.12 & -0.18 & 0.12 & 0.55 & -0.40 & 2.96 \\
\hline C 4-1 & 9.69 & 0.07 & -0.16 & 0.12 & 0.62 & -0.32 & 3.79 \\
\hline C $13-3$ & 10.58 & 0.60 & 0.26 & 0.32 & 0.49 & 0.08 & 6.69 \\
\hline C $13-1$ & 10.55 & 0.61 & 0.08 & 0.31 & 0.64 & -0.25 & 6.46 \\
\hline C $14-2$ & 9.75 & -0.20 & -0.37 & 0.12 & 0.23 & -0.21 & 3.82 \\
\hline C $20-2$ & 9.93 & -0.12 & -0.43 & 0.13 & -0.09 & -0.47 & 3.05 \\
\hline C 21-1 & 10.49 & 0.60 & 0.29 & 0.28 & 0.07 & -0.12 & 2.50 \\
\hline C $22-2$ & 10.18 & 0.69 & 0.43 & 0.17 & 0.46 & 0.06 & 3.67 \\
\hline D 0-2 & 10.39 & 0.99 & 0.81 & 0.24 & 1.01 & 0.64 & 2.83 \\
\hline D $10-4$ & 9.74 & 0.73 & 0.58 & 0.12 & 0.91 & 0.42 & 2.22 \\
\hline D 13-1 & 9.23 & 0.11 & -0.19 & 0.10 & 0.35 & -0.17 & 3.14 \\
\hline D $13-5$ & 10.73 & 1.18 & 0.89 & 0.38 & 1.09 & 0.72 & 4.67 \\
\hline D 14-1 & 10.31 & 0.81 & 0.51 & 0.21 & 0.84 & 0.35 & 4.05 \\
\hline D $15-1$ & 9.70 & -0.02 & -0.21 & 0.12 & 0.19 & -0.10 & 2.55 \\
\hline D $15-2$ & 9.28 & -0.12 & -0.25 & 0.10 & -0.22 & -0.33 & 1.58 \\
\hline D $15-3$ & 10.73 & 0.89 & 0.77 & 0.38 & 0.86 & 0.35 & 5.73 \\
\hline D $20-1$ & 10.27 & 0.75 & 0.27 & 0.20 & 0.50 & 0.18 & 3.17 \\
\hline D 21-3 & 10.48 & 0.79 & 0.44 & 0.28 & 0.26 & 0.07 & 4.83 \\
\hline D 22-1 & 10.77 & 0.83 & 0.57 & 0.40 & 0.54 & 0.06 & 3.12 \\
\hline D $22-2$ & 9.97 & 0.71 & 0.56 & 0.13 & 0.73 & 0.29 & 4.55 \\
\hline D 23-1 & 10.01 & 0.58 & 0.35 & 0.13 & 0.66 & 0.37 & 2.80 \\
\hline E 0-3 & 10.72 & 0.84 & 0.47 & 0.37 & 0.70 & 0.33 & 6.05 \\
\hline E $0-2$ & 10.47 & 1.03 & 0.72 & 0.27 & 0.75 & 0.64 & 2.22 \\
\hline E 4-1 & 10.77 & 1.01 & 0.90 & 0.39 & 0.87 & 0.64 & 2.56 \\
\hline E 9-1 & 10.56 & 0.49 & 0.49 & 0.32 & 0.65 & 0.26 & 3.45 \\
\hline E $10-1$ & 10.81 & 1.45 & 1.38 & 0.41 & 1.07 & 0.70 & 2.79 \\
\hline E 23-1 & 10.30 & 0.69 & 0.60 & 0.21 & 0.95 & 0.67 & 1.67 \\
\hline F 8-2 & 9.87 & 0.93 & 0.74 & 0.12 & 1.36 & 0.74 & 1.78 \\
\hline F 9-1 & 10.36 & 1.40 & 1.21 & 0.23 & 1.90 & 1.12 & 3.41 \\
\hline F $10-1$ & 9.61 & 0.30 & 0.30 & 0.12 & 0.81 & 0.30 & 1.62 \\
\hline F $12-4$ & 10.18 & 0.95 & 0.36 & 0.16 & 1.28 & 0.41 & 2.75 \\
\hline G 3-2 & 9.81 & 0.90 & 0.81 & 0.12 & 0.98 & 0.66 & 2.38 \\
\hline G 3-4 & 10.81 & 1.61 & 1.52 & 0.41 & 1.45 & 1.22 & 2.48 \\
\hline G 4-1 & 10.81 & 1.45 & 1.29 & 0.41 & 1.30 & 0.99 & 2.44 \\
\hline G 8-4 & 10.04 & 0.75 & 0.63 & 0.14 & 0.68 & 0.55 & 1.94 \\
\hline G 8-5 & 10.24 & 0.99 & 0.92 & 0.19 & 0.99 & 0.84 & 2.30 \\
\hline G 8-1 & 9.90 & 0.78 & 0.71 & 0.13 & 0.76 & 0.51 & 1.77 \\
\hline G 8-2 & 10.61 & 1.28 & 1.26 & 0.33 & 1.16 & 0.94 & 1.81 \\
\hline G 8-3 & 10.41 & 1.02 & 1.02 & 0.25 & 1.34 & 0.95 & 2.41 \\
\hline G 9-1 & 10.42 & 1.08 & 1.08 & 0.25 & 1.16 & 0.97 & 1.92 \\
\hline G $10-1$ & 10.09 & 0.98 & 0.98 & 0.14 & 1.20 & 0.89 & 1.89 \\
\hline G 11-1 & 10.70 & 1.31 & 1.31 & 0.37 & 1.34 & 0.93 & 3.01 \\
\hline G 13-1 & 10.05 & 1.39 & 1.34 & 0.14 & 1.25 & 1.03 & 2.01 \\
\hline G $14-1$ & 10.35 & 1.00 & 0.91 & 0.23 & 0.75 & 0.65 & 1.85 \\
\hline
\end{tabular}


Table 1 - continued

\begin{tabular}{|c|c|c|c|c|c|c|c|}
\hline \multirow[t]{2}{*}{ Galaxy ID } & \multirow[t]{2}{*}{$\begin{array}{c}\text { Stellar mass } \\
\left(\log \mathrm{M}_{\odot}\right)\end{array}$} & \multicolumn{3}{|c|}{$\begin{array}{c}\mathrm{SFR}_{\mathrm{B} 04} \\
\left(\log \mathrm{M}_{\odot} \mathrm{yr}^{-1}\right)\end{array}$} & \multicolumn{2}{|c|}{$\begin{array}{c}\mathrm{SFR}_{\mathrm{IFS}} \\
\left(\log \mathrm{M}_{\odot} \mathrm{yr}^{-1}\right)\end{array}$} & \multirow[t]{2}{*}{$\gamma(u$ band $)$} \\
\hline & & Total & Fibre & Correction & Total & Fibre & \\
\hline G $14-3$ & 10.42 & 1.30 & 1.26 & 0.25 & 1.17 & 1.08 & 1.60 \\
\hline G 20-1 & 10.72 & 1.43 & 1.29 & 0.37 & 1.46 & 1.31 & 1.81 \\
\hline G 20-2 & 10.33 & 1.28 & 0.93 & 0.22 & 1.03 & 0.86 & 1.80 \\
\hline G $21-2$ & 10.03 & 0.99 & 0.80 & 0.13 & 1.11 & 0.81 & 2.03 \\
\hline H $10-2$ & 9.98 & 1.10 & 0.83 & 0.13 & 1.40 & 0.83 & 1.91 \\
\hline
\end{tabular}

\section{CORRECTING FOR MISSING LIGHT IN ESTIMATES OF STAR FORMATION}

There are two basic methods for correcting measurements for the incomplete coverage of the fibre spectrum. On the one hand, the measurement can simply be scaled up to account for incomplete coverage. Scaling factors used are typically based on imaging data, where the ratio of fibre-to-galaxy size or fibre-to-galaxy luminosity can be measured. The quality of the correction will depend on how well the chosen imaging bandpass acts as a proxy for the measurement of interest. On the other hand, the missing information can be reconstructed using additional data and more complex methods. A common example is to combine scaling with colour information from imaging in multiple bands.

Of the two correction methods, which is best will depend on the measurement of interest. Measurements of star formation can reasonably be corrected using the scaling approach (by assuming that the distribution of star formation follows that of broad-band light). However, measurements of metallicity, where the distribution is unlikely to match that of broad-band light, require a reconstructive method. Regardless of correction method, the assumptions made must be carefully examined and tested. Reconstructive methods can often be tested or calibrated using regions covered by the fibre spectroscopy. Scaling methods can be tested using similar classes of objects at different distances (such that the extent of the fibre coverage varies) or by changing the size of the aperture e.g. by dithering. Below we review aperture corrections for SFR using both of these methods.

For simplicity, we define the aperture correction, $\gamma$, as the ratio of the total-to-aperture quantity for a particular observable, such as luminosity,

$\gamma(L) \equiv \frac{L_{\text {total }}}{L_{\text {aperture }}}$.

We use $\gamma$ regardless of which correction method is used, and also for directly measured aperture corrections discussed later.

\subsection{Example of a scalar aperture correction}

Gilbank et al. (2010) use a scalar aperture correction to estimate the total SFR for SDSS galaxies. They scale the SFR by the ratio of $u$-band flux within the fibre to the total (Petrosian) $u$-band flux. We refer to this scaling as $\gamma$ ( $u$-band). The scaling is done independently of the determination of the SFR within the fibre. They argue that $u$-band is most sensitive to young blue stars associated with recent and ongoing star formation, and therefore best for the scaling. They do note, however, that both $g$ - and $r$-band scaling give similar results for all but the largest mass galaxies. No differential dust correction for the annular region outside the fibre is directly included.
To check the accuracy of the scalar aperture correction, Gilbank et al. (2010) compare their aperture-corrected measurements with alternative indicators of star formation that do not require correction. SFRs derived using both $u$-band photometry and far-ultraviolet (FUV) photometry agree reasonably with the aperture-corrected SFRs across the full range in stellar masses observed.

\subsection{Example of a reconstructive aperture correction}

Brinchmann et al. (2004) use a reconstructive aperture correction to estimate the total SFR for SDSS galaxies. Their procedure maps ${ }^{0.1} i$-band luminosity, $L_{i}$, and two colours, ${ }^{0.1}(g-r)$ and ${ }^{0.1}(r-i)$, to SFR as follows. Star formation rate scales with $i$-band luminosity, $\eta_{i}=L_{i} / \mathrm{SFR}$, but $\eta_{i}$ depends on colour. A likelihood function $P\left(\eta_{i} \mid\right.$ colour $)$ provides the mapping. $P\left(\eta_{i} \mid\right.$ colour $)$ is determined empirically using the information available within the fibre aperture for the whole of the star-forming SDSS sample, and then used to reconstruct the star formation outside the fibre for each galaxy. This annular star formation is added to the central star formation measured using the fibre spectra to determine the total SFR of the galaxy. For our purposes, we define $\gamma\left(\mathrm{SFR}_{\mathrm{B} 04}\right)$ as the ratio of their total SFR to their fibre SFR.

Even though the reconstruction is calibrated using the regions of the galaxies covered by the fibres, it is simultaneously possible to determine the expected accuracy of the reconstruction. The calibration is formed by marginalizing over the (large) training set, providing a full probability distribution for each colour and luminosity of the reconstruction. For some colours, the probability distribution of $\eta_{i}$ is quite large, providing no better than an order-of-magnitude constraint on the reconstructed SFR. However, because the reconstruction only contributes to part of the final measurement of SFR, this weak constraint may not dominate the error of the combined measurement.

\subsection{Testing aperture-correction methods with measured aperture ratios}

Using our integral-field spectroscopic data, we can directly measure an aperture correction and compare with the inferred corrections discussed above. From our IFS data, we measure $\gamma\left(L_{\mathrm{H} \alpha}\right)$ as the ratio of the total $\mathrm{H} \alpha$ luminosity to the luminosity within a synthetic aperture matched to the size and location of the SDSS fibre. Fig. 2 compares our $\gamma\left(L_{\mathrm{H} \alpha}\right)$ with both the (estimated) ratio for SFR of Brinchmann et al. (2004), $\gamma\left(\mathrm{SFR}_{\mathrm{B} 04}\right)$, and the (directly measured) $u$-band ratio, $\gamma$ ( $u$-band), used to determine total SFRs by Gilbank et al. (2010). The correlations between the two correction methods and the actual $\gamma\left(L_{\mathrm{H} \alpha}\right)$ are present but marginal; Pearson's $R=0.54$ for Brinchmann et al. and Gilbank et al. ( $R$ is measured in the logarithmic space). 
A test of SDSS aperture corrections with IFS
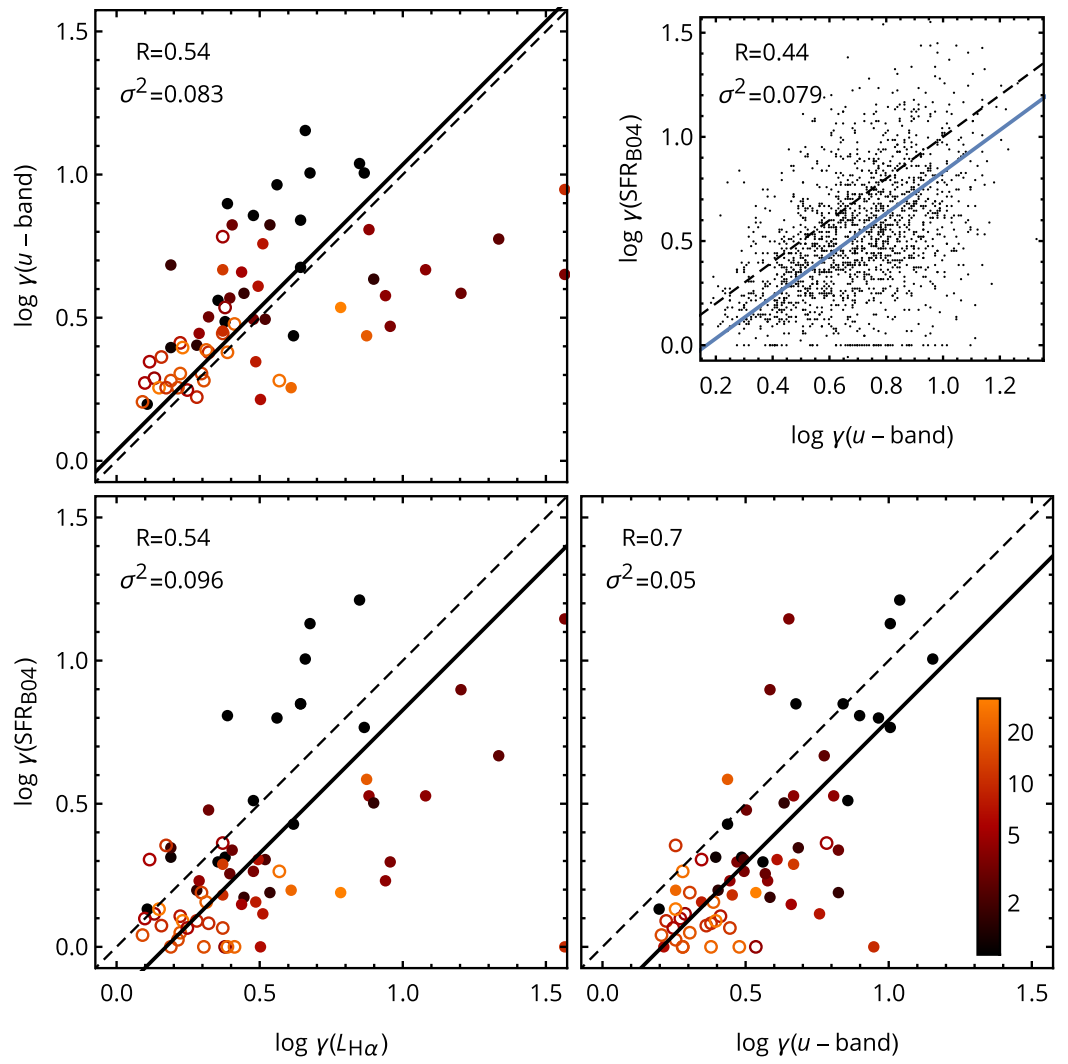

Figure 2. Comparison of aperture corrections with the directly measured $\mathrm{H} \alpha$ aperture ratio, $\gamma_{\mathrm{H} \alpha}$. The three large panels show the relationships between the

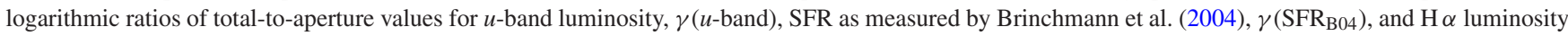
as measured here, $\gamma\left(L_{\mathrm{H} \alpha}\right)$. The solid line in each panel shows the best-fitting systematic offset (a line with slope of unity and fitted intercept). Round symbols are in the redshift range $0.055<z<0.084$, square symbols are at higher redshift. The symbols are colour coded for their SFRs according to the colour bar in the bottom right. Also shown in each panel is the Pearson's correlation coefficient, $R$, and the variance around the line, $\sigma^{2}$. The inset panel shows the correlation between $\gamma\left(\mathrm{SFR}_{\mathrm{B} 04}\right)$ and $\gamma(u$-band) for star-forming SDSS galaxies in the redshift range $0.055<z<0.084$.

We have also reviewed the correlation between aperture corrections in Brinchmann et al. (2004) and Gilbank et al. (2010) more generally. The inset panel in Fig. 2 shows the relationship between $\gamma(u$-band $)$ and $\gamma\left(\mathrm{SFR}_{\mathrm{B} 04}\right)$ for 1705 star-forming galaxies in the redshift range $0.055<z<0.084$ (matching our IFS sample), and right ascension range $0^{\circ}-40^{\circ}$. For this larger sample, the correlation between the two aperture-correction methods is poorer, $R=0.44$. This weak correlation is not inconsistent with Gilbank et al. - we are considering only a very restricted redshift range. In fact, the standard deviation of the scatter measured in this restricted range, $\sigma=0.28 \mathrm{dex}$, is smaller than the $\simeq 1 \mathrm{dex}$ reported for the whole of Stripe 82 by Gilbank et al. (see their fig. A2). We see the same offset they identified, namely $\gamma(u$-band $)$ is $\simeq 0.2$ dex larger than $\gamma\left(\mathrm{SFR}_{\mathrm{B} 04}\right)$.

The scatter of the aperture corrections can be used to determine the variation in the SFRs derived using them (over the range of SFRs, $0.1-100 \mathrm{M}_{\odot} \mathrm{yr}^{-1}$ in our sample). The scatter in the $u$ band correction against the directly measured $\mathrm{H} \alpha$ correction corresponds to a standard deviation of $\sigma=0.29 \mathrm{dex}$ in SFRs corrected with $\gamma$ (u-band). The correction $\gamma(u$-band) does not systematically over- or underestimate $\gamma(\mathrm{H} \alpha)$, and nor the total SFR (observed offset of $0.04 \mathrm{dex})$. The correction $\gamma\left(\mathrm{SFR}_{\mathrm{B} 04}\right)$ introduces a scatter of \pm 0.31 dex in the SFRs, and a very mild systematic underestimate of 0.17 dex. Both aperture-correction approaches introduce scat-
Table 2. Impact of aperture corrections on SFRs.

\begin{tabular}{lcc}
\hline $\begin{array}{l}\text { Aperture } \\
\text { correction }\end{array}$ & $\begin{array}{c}\text { Additional } \\
\text { scatter } \\
(\text { dex })\end{array}$ & $\begin{array}{c}\text { Systematic } \\
\text { offset } \\
(\text { dex })\end{array}$ \\
\hline$u$ band & 0.29 & 0.04 \\
Brinchmann et al. (2004) & 0.31 & -0.17 \\
\hline
\end{tabular}

ter over the directly measured $\mathrm{H} \alpha$ aperture correction, necessarily weakening the statistical power in estimates of SFR. These results are summarized in Table 2.

The $\mathrm{H} \alpha$ correction measured here is systematically larger than $\gamma\left(\mathrm{SFR}_{\mathrm{B} 04}\right)$, but agrees well with $\gamma\left(u\right.$-band). The $\mathrm{SFR}_{\mathrm{B} 04}$ ratio is within one standard deviations of $\gamma(\mathrm{H} \alpha)$. The differences are also consistent with the $\simeq 0.2$ dex already identified between $\gamma\left(\mathrm{SFR}_{\mathrm{B} 04}\right)$ and $\gamma$ ( $u$-band) by Gilbank et al. (2010). While Gilbank et al. have no explanation for this $\sim 0.2$ dex difference, it vanishes in the star formation rate densities ostensibly because of the different derivation of SFRs.

Different methods for determining the total flux ( $u$-band, $\mathrm{H} \alpha$ or B04 SFR) could also explain the offsets observed. Even without complications of a fibre aperture, choosing an aperture/method for determining galaxy photometry is complicated. Brinchmann et al. 
A test of SDSS aperture corrections with IFS
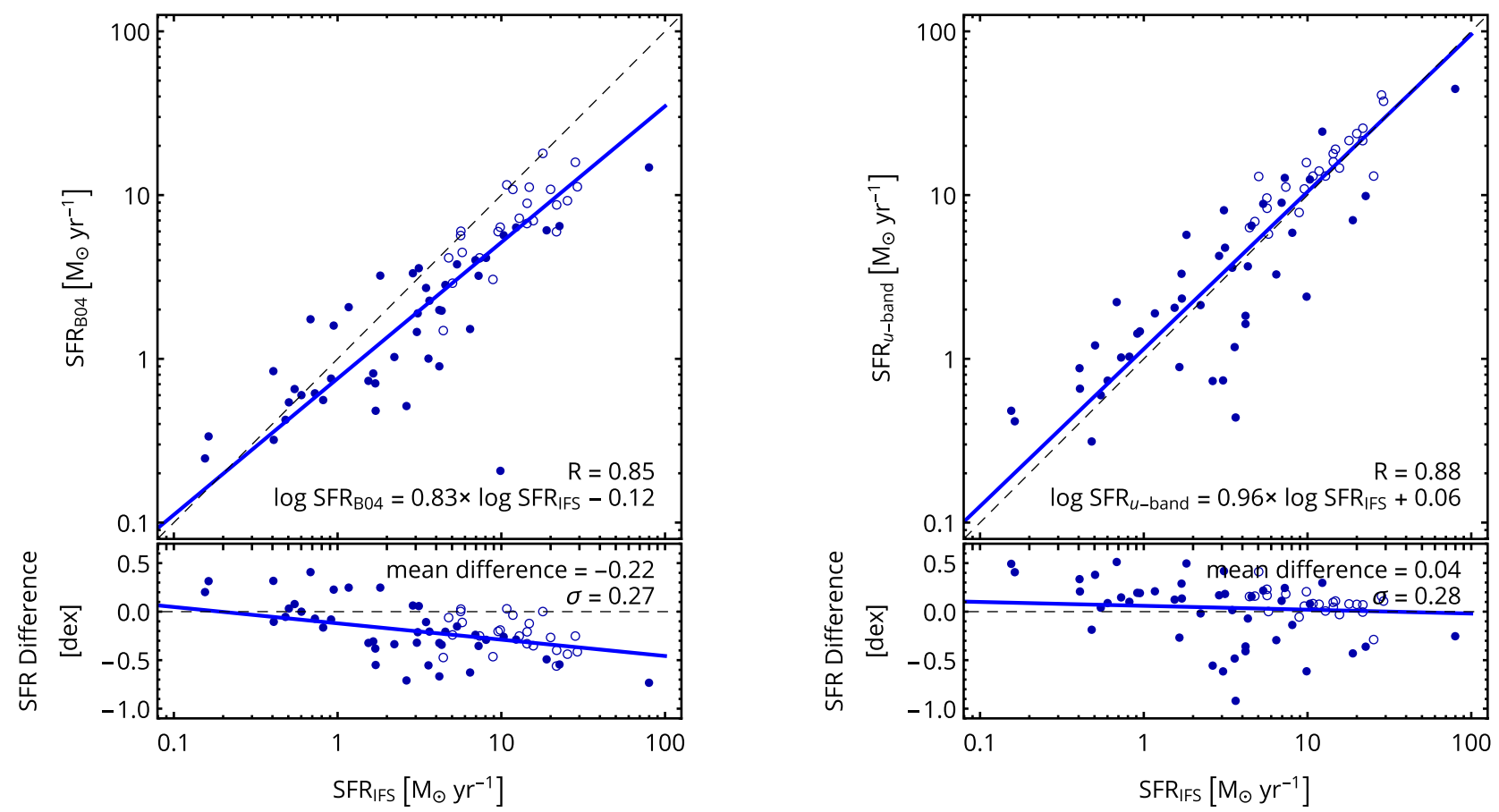

Figure 3. The aperture-corrected SFRs of Brinchmann et al. (2004), SFR $\mathrm{B}_{04}$ (left) and $u$-band corrections based on Gilbank et al. (2010) (right) compared with the total SFRs measured via IFS (blue symbols). The filled symbols show galaxies with $0.055<z<0.084$, open symbols are galaxies at higher redshift. The $\mathrm{SFR}_{\mathrm{B} 04}$ rates have been adjusted to match our estimates of SFR as described in Section 2.2 (the comparison without correction recovers a similar slope). The solid blue line shows the best fit to the relationship, with equation as shown. Also shown is the Pearson's correlation coefficient, $R$. Bottom panels show the residual between the two estimates, with the mean offset and scatter, $\sigma$, about the best-fitting line printed. A systematic underestimate of star formation in higher star formation rate galaxies is apparent in Brinchmann et al. The same problem is formally present, but less extreme for $u$-band corrections. In all panels, the dashed lines show the one-to-one relationship.

(2004) use SDSS 'cmodel' magnitudes in the determination of total SFR, while Gilbank et al. (2010) use SDSS Petrosian magnitudes and DYNAMO uses a flux limit. Systematic differences between these methods would lead to systematic differences in the aperture ratios. Since SDSS Data Release 2, there is excellent agreement between 'cmodel' and Petrosian magnitudes, but with a systematic offset of 0.05-0.1 mag for bright galaxies. ${ }^{3}$ An offset of 0.1 mag is not enough to explain a change in aperture ratio of $0.1 \mathrm{dex}$, which would require a luminosity change of $0.25 \mathrm{mag}$. The light missed by our IFS (typically less than 10 per cent) is also too small to account for such a large change in aperture ratio. Therefore, it seems unlikely that differences in total flux determinations could explain the systematic differences in $\gamma$ seen here.

With spatially resolved dust maps, one might explain the offsets in the different aperture ratios more completely, but such data are not available to us.

\subsection{Aperture corrections and total rates of star formation}

The aperture-corrected SFRs do correlate well with the measured total SFRs, despite the marginal correlation of aperture-correction estimates with the actual aperture loss. Fig. 3 shows how two different aperture-corrected SFRs correlate with total star formation from our IFS data. The fits shown in this figure, and Fig. 4 below, are done using a linear regression method that is symmet-

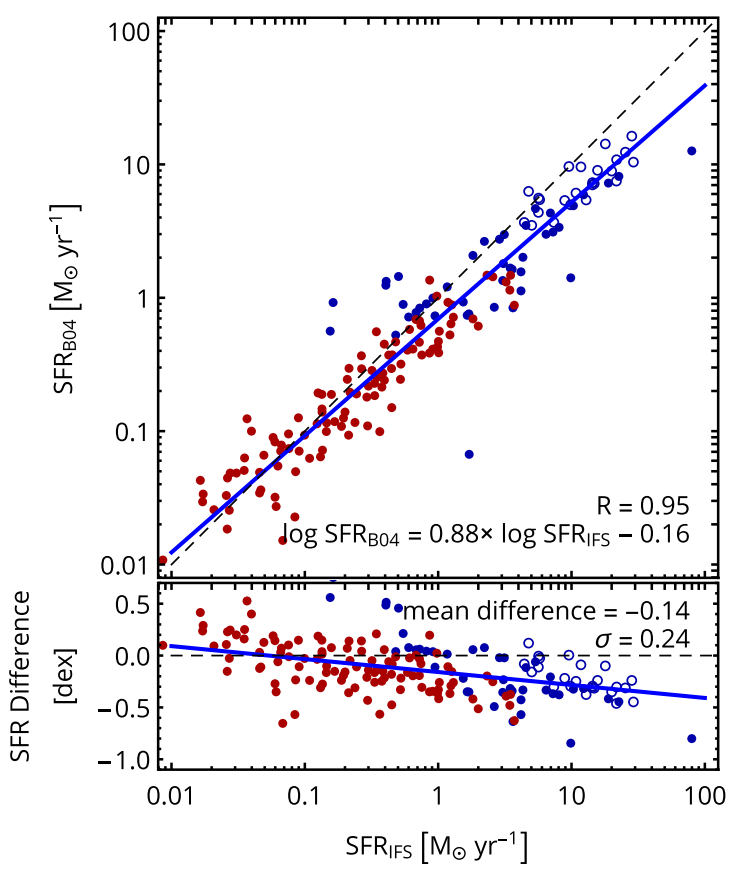

Figure 4. Combined comparison of IFS and aperture SFRs as for the left half of Fig. 3. Grey points (red online) are drawn from Richards et al. (2016a), black (blue online) symbols are from this work as before.

\footnotetext{
${ }^{3}$ http://classic.sdss.org/dr4/algorithms/photometry.html\#mag_petro
} 
ric, i.e. the choice of dependent variable does not affect the fit (HYPER FIT; ${ }^{4}$ Robotham \& Obreschkow 2015). The left-hand panel shows the comparison for rates from Brinchmann et al. (2004) The right-hand panel shows the comparison for SFRs derived by applying a $u$-band aperture correction similar to that of Gilbank et al. (2010). Both aperture-loss correction methods show a stronger correlation with those from IFS than might be expected from the weak correlation in aperture-correction ratios explored earlier. The correlation is stronger because aperture corrections tend to be less than a factor of 10 (true for $>90$ per cent of galaxies considered here), while the range in SFRs in the sample is a factor of $\sim 1000$, so the comparison is dominated by the range in star formation, not the scatter in aperture correction.

We have adjusted the SFRs of Brinchmann et al. (2004) as noted earlier to ensure Fig. 3 provides a direct test of aperture corrections and not of the method of determining SFR. Brinchmann et al. use a different method to determine SFRs than our simpler Kennicutt scaling (see Sections 2.1 and 2.2). To bring their SFR estimation method in line with ours (ours being more common in the literature), we adjust their rates according to the empirical correction provided in their fig. 8. The size of this correction is shown in the column labelled 'Correction' of Table 1 . The net effect of this correction on Fig. 3 is to shift points down by 0.21 dex and decrease the slope from 0.94 to 0.83 . When comparing our results with Richards et al. (2016a), note that they do not make such a correction to the Brinchmann et al. SFRs; we make this comparison carefully in Section 3.5. The adjustment of Brinchmann et al. SFRs has been made purely for a more direct test of aperture corrections.

In general, the aperture-corrected SFRs of Brinchmann et al. (2004) correlate well with results from IFS $(R=0.85$ in logarithmic space), but individual galaxies do scatter above and below the one-to-one relation by $\pm 0.27 \mathrm{dex}$. The correlation is similar for $u$-band-corrected rates, with $R=0.88$ and scatter of $\pm 0.28 \mathrm{dex}$. The best-fitting line to the correlation for each shows an underestimate in aperture-corrected SFR that grows with total SFR. This systematic underestimate is stronger for Brinchmann et al. aperture-corrected rates. Richards et al. (2016a) find the same effect, but with a sample of galaxies at lower SFRs than considered here. Despite good correlation, aperture-corrected SFRs systematically underestimate star formation in highly star-forming galaxies, especially when using the correction method of Brinchmann et al.

\subsection{The complementary data of Richards et al.}

Our sample probes high rates of star formation, $\sim 1-100 \mathrm{M}_{\odot} \mathrm{yr}^{-1}$, but the Richards et al. (2016a) sample probes a lower range of SFRs, $0.01-\simeq 5 \mathrm{M}_{\odot} \mathrm{yr}^{-1}$. The two samples are therefore very complementary. The two samples are combined here to better understand where aperture losses may be over- or underestimated.

To combine the data fairly, we have (1) scaled the Richards et al. (2016a) SFRs and stellar masses by a factor of 0.56 to account for their choice of a Salpeter IMF; (2) retrieved DR7 values of the SFRs and stellar masses for our galaxies to match the choice of Richards et al. (2016a) ${ }^{5}$ and (3) applied the same methodology correction to the $\mathrm{SFR}_{\mathrm{B} 04}$ for both samples (described in Section 2.2). One difference remains between the estimates of star formation: Richards et al. (2016a) apply a dust correction derived using a total

\footnotetext{
${ }^{4}$ HYPER FIT is available online: http://hyperfit.icrar.org/

5 see the appendix for a more detailed discussion of how the choice of DR4 versus DR7 largely does not affect our results
}
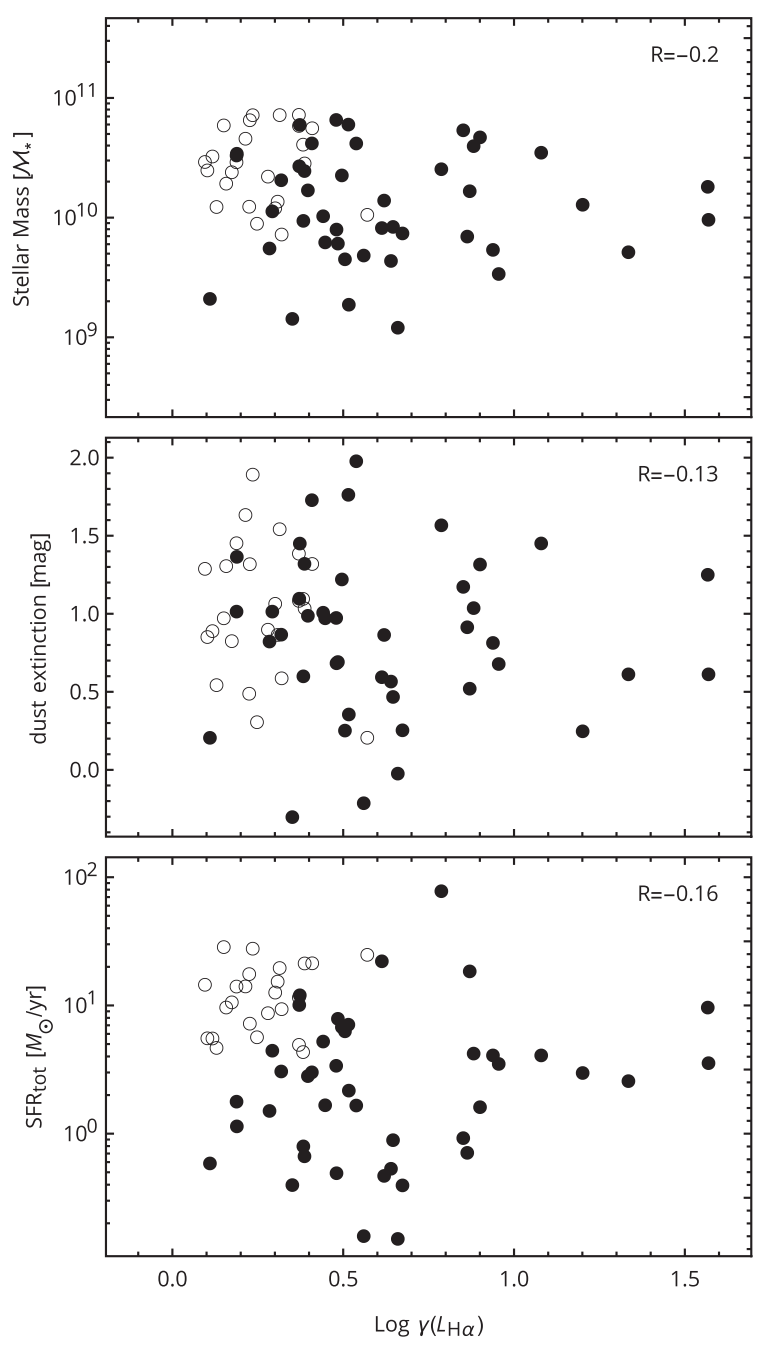

Figure 5. No correlation is apparent between the directly measured $\mathrm{H} \alpha$ aperture loss and either galaxy stellar mass (top), dust extinction at $\mathrm{H} \alpha$ (middle) or SFR (bottom). The Pearson's $R$ correlation coefficient for each comparison is shown. Solid symbols show galaxies in the redshift range $0.055<z<0.84$, open symbols are galaxies at higher redshifts.

spectrum instead of the 3 -arcsec central aperture spectrum. Their correction is still not spatially resolved.

Remarkably, the results for the combined data are largely the same as the results for the individual data sets. Fig. 4 shows the combined data (same format as the left-hand panel of Fig. 3). In the combined plot, the correlation coefficient improves to 0.95 . The best-fitting line for the combined data set has a slope closer to unity, 0.88 , but still suggesting a systematic difference from Brinchmann et al. (2004). A similar systematic is also seen in just the Brinchmann et al. data. The scatter decreases slightly in the combined data to 0.24 dex. These similarities provide independent support for our results.

\subsection{Other systematic effects on aperture corrections}

Now we discuss what systematic effects are apparent in the aperture corrections with respect to dust, stellar mass and total SFR.

Our data do not support a correlation of the size of the $\mathrm{H} \alpha$ aperture correction, $\gamma\left(L_{\mathrm{H} \alpha}\right)$, with the stellar mass. The comparison is shown in Fig. 5. Galaxies in our sample have stellar masses of $10^{9}-10^{11}$ 
as determined by Kauffmann et al. (2003). We see no systematic correlation (Pearson's $R=-0.20$ measured in logarithmic space). However, Gilbank et al. (2010) see a weak but significant correlation of $\gamma$ ( $u$-band) with stellar mass, with the most massive galaxies $\left(\log \mathcal{M}_{*}>10.8\right.$ ) having an $\sim 0.1$ dex larger $\gamma(u$-band) at $z \approx 0.05$. The difference shrinks at higher redshifts. We conclude that a larger sample is need to determine if stellar mass has a systematic effect on aperture corrections for SFRs in SDSS.

There is also no correlation $(R=-0.13)$ of $\gamma(\mathrm{H} \alpha)$ with dust attenuation determined by the Balmer decrement method, $A_{\mathrm{H} \alpha}$, BD (within the fibre aperture), nor with SFR $(R=-0.16)$. These are both also shown in Fig. 5. Again, this lack of correlation is limited by our sample size. However, there is perhaps a very weak correlation (not shown) between aperture correction and the apparent, photometric $r$-band size of the galaxy $(R=0.33)$ in our data, which is as one would expect.

\subsection{Variable extinction}

For our star formation rate estimates, we are unable to apply a spatially varying dust-extinction correction using the Balmer decrement method. Instead, a fixed correction based on the $\mathrm{H} \alpha / \mathrm{H} \beta$ ratio within the fibre has been applied. The method of Brinchmann et al. (2004) can account for a variable dust extinction, at least in principle. The lack of a spatially varying dust correction is a weakness of our analysis, which we address in this section.

Kewley et al. (2005) have used the Nearby Field Galaxy Survey to investigate if the central dust extinction differs significantly from the total dust extinction. That survey includes both nuclear and integrated spectra. Although there is some scatter in individual galaxies, they find that the differences in extinction between the nuclear and integrated spectra are consistent with zero. They also see no significant deviation from zero when the sample is subdivided by galaxy type. This finding implies our dust-correction approach will not affect the SFRs.

Flat extinction gradients in galaxies have also been observed in the sample of Kennicutt, Keel \& Blaha (1989) and for M83 by Kaufman et al. (1987). However, studies of M101 and M33 have shown extinction gradients in those galaxies, although these extinction estimates may differ from those calculated using the Balmer decrement (section 5 of Kewley et al. 2005, and references therein). Furthermore, extinction gradients have been identified in six galaxies by Boissier et al. (2004), though it is worth noting that many of their galaxies for which extinction has been measured using the Balmer decrement method for $\mathrm{H}$ II regions also show considerable scatter among individual measurements, often as large or larger than the measured gradient.

A paper by Wild et al. (2011) provides an analysis of dust properties in a much larger sample of 23000 galaxies. This analysis shows radial gradients in dust attenuation of stellar continuum emission: stellar continuum emission is more strongly attenuated in the centres of galaxies than in the outer regions, and this difference is more pronounced in galaxies with higher specific SFRs. Their analysis cannot test directly for the same effect on emission from starforming $\mathrm{H}$ II regions because it lacks the necessary data. However, Wild et al. are able to probe how continuum and Balmer decrement dust measurements relate, and find the relation to also be a strong function of specific SFR. From this analysis, it is probable, but not demonstrated, that dust will more strongly attenuate star formation indicators in the central regions of galaxies than the outer regions, which would cause our analysis to overpredict the total SFR for each galaxies.
Richards et al. (2016a) provide two pieces of insight into the possible impact of dust on our results. First, they provide some information on the relative impact of dust in the nucleus and disc. Their fig. 11 shows the nuclear-to-disc ratio of the Balmer decrement for 337 star-forming SAMI galaxies. They find a weak correlation where galaxies with higher SFRs tend to have dustier nuclei. ${ }^{6}$ This finding implies our dust-correction approach will overpredict the SFRs. The second piece of insight is a test of whether Brinchmann et al. (2004) can account for a variable dust extinction. Richards et al. (2016a) repeat the aperture correction both using the nuclear spectra (as is used for Brinchmann et al.) and the disc spectra. Their analysis reveals that a nuclear-based aperture correction overpredicts the SFR of the disc compared with a disc-based aperture correction when the nucleus is dustier than the disc. ${ }^{7}$ These two insights imply that both Brinchmann et al. and our own analysis will overpredict disc star formation in highly star-forming galaxies.

We have tested for the possibility of varying dust extinction using equivalent widths of $\mathrm{H} \alpha$ and ${ }^{0.1}(g-r)$ colours for our galaxies. These two parameters: are independent approximate measures of the specific SFR (as both are sensitive to the mix of young and old stars); are available inside and outside our fibre apertures and have different sensitivities to dust. There is no visual evidence for a difference in distribution, nor does a 2D Peacock-KolmogorovSmirnov test (Peacock 1983) provide any evidence for a difference in the distributions (the maximum absolute difference calculated via that test is 0.05 out of 1 ).

Furthermore, we note that when we correct the $\mathrm{H} \alpha,{ }^{0.1}(g-r)$ distribution for dust using a Calzetti et al. (2000) attenuation law for continuum, a standard reddening curve for the nebular lines (Pei 1992) and the SDSS extinction measure, we find both distributions are consistent with a Salpeter IMF slope at the high-mass end for the stellar population (Hoversten \& Glazebrook 2008).

Considering the various analyses and their results presented above, it remains unclear just how much our total SFRs might be affected by our method of correction for dust attenuation. We admit that our choice to extrapolate a central estimate of dust correction to the whole of each galaxy could introduce biases that would affect our final results. However, Brinchmann et al. (2004) SFRs are biased for similar reasons, so there may in fact be little change to our finding of an underestimate in that work. A dust extinction estimate from the whole of each galaxy is better (this is the approach of Richards et al. 2016b), but a spatially resolved, Balmer decrement method, dust-attenuation correction for analyses such as ours would be more satisfying, and should be pursued in future.

\subsection{Impact on the local density of star formation}

If total SFRs are systematically biased as we have found, we consider as an experiment what the impact is on the total density of star formation. We take the luminosity function data from Gilbank et al. (2010) and adjust it according to the underestimate found for Brinchmann et al. (2004) SFRs in Fig. 3. We scale up the Balmerdecrement-correctedSFRs, $\mathrm{SFR}_{\mathrm{Gil}}$ as follows:

$$
\log \mathrm{SFR}=\frac{1}{0.83}\left(\log \mathrm{SFR}_{\mathrm{Gil}}-0.12\right) .
$$

We then recompute, following the same method, the global SFR density of the Universe locally $(0.032<z<0.20)$. The density

\footnotetext{
${ }^{6}$ Note that the Richards et al. (2016a) paper's original text was incorrect on this point, see the erratum Richards et al. (2016b).

${ }^{7}$ Again, the original text is corrected in the erratum Richards et al. (2016b).
} 
Table 3. Measurements of the local star formation rate density.

\begin{tabular}{lcc}
\hline Reference & Method $^{a}$ & SFRD $^{b}$ \\
\hline Brinchmann et al. (2004) & Charlot et al. (2002) model grid & 16.9 \\
Sadler et al. (2002) & $1.4 \mathrm{GHz}$ & 10.4 \\
Sullivan et al. (2000) & 2000 & 21.9 \\
Gilbank et al. (2010) & $\mathrm{H} \alpha$ Balmer decrement & 12.1 \\
- with systematic SFR correction from Section 3.8 & 22.5 \\
- with combined DYNAMO and SAMI & 10.2 \\
\hline
\end{tabular}

Notes. ${ }^{a}$ Wavelength and/or method of estimate.

${ }^{b}$ The SFR density measured in units of $10^{-3} \mathrm{M}_{\odot} \mathrm{yr}^{-1} \mathrm{Mpc}^{-3}$. Values converted from Kroupa (2001) IMF using factor of 0.88, and from Salpeter (1955) IMF using factor of 0.56 .

${ }^{c}$ As reported by Hopkins (2004).

remains the same, $12.1 \times 10^{-3} \mathrm{M}_{\odot} \mathrm{yr}^{-1} \mathrm{Mpc}^{-3}$. If instead, we use a correction derived from both our data and that of Richards et al. (2016a), the density decreases to $9.7 \times 10^{-3} \mathrm{M}_{\odot} \mathrm{yr}^{-1} \mathrm{Mpc}^{-3}$.

The star formation rate density of the Universe has been measured in many different ways, giving a range of estimates of 10-22 $\times$ $10^{-3} \mathrm{M}_{\odot} \mathrm{yr}^{-1} \mathrm{Mpc}^{-3}$ (see Table 3; Hopkins 2004). These results may seem to suggest that measurements of the density are fairly robust to biases introduced by aperture effects. However, we note that they are actually quite sensitive to such biases: using a slightly steeper slope found for DR7 results (discussed in Appendix A) causes the density to double to $20.6 \times 10^{-3} \mathrm{M}_{\odot} \mathrm{yr}^{-1} \mathrm{Mpc}^{-3}$. This experiment has highlighted to us the difficulties inherit in developing a statistical picture of the Universe when corrections for aperture losses play a role in the measurements used.

\section{CONCLUSIONS AND DISCUSSION}

We have used spatially resolved measurements of $\mathrm{H} \alpha$ emission to find both the total SFR and to directly measure the aperture correction required for an SDSS fibre aperture. These measurements have been compared with other estimates of the total star formation and the corrections for aperture losses used in those estimates. Our main findings are as follows:

(i) Aperture-correction methods tend to underestimate SFRs for galaxies with rates of $1-100 \mathrm{M}_{\odot} \mathrm{yr}^{-1}$ in SDSS (similar to the findings of Gerssen et al. 2012), with the most commonly cited rates, those of Brinchmann et al. (2004), underestimated by 0.17 dex for the sample considered here (Fig. 2 and Table 2).

(ii) The underestimate of SFR by Brinchmann et al. (2004) is larger for galaxies with higher SFRs (Fig. 3), in agreement with Chang et al. (2015) and Richards et al. (2016a).

(iii) Estimates of $\mathrm{H} \alpha$ aperture losses for individual galaxies only correlate marginally with the true aperture loss, and these estimates vary by a factor of several in most cases (Fig. 2).

(iv) Despite the large variation, aperture-loss estimates represent only a second-order effect in measurements of the total SFRs of galaxies.

(v) Estimates of the local SFR density of the Universe can be sensitive to biases in corrections for aperture losses leading to a factor of 2 variation in those estimates.

(vi) These conclusions are limited by the lack of a spatially resolved dust-attenuation correction in our analysis, and there is evidence both ways as to whether this omission adversely affects our results, but the SFRs of Brinchmann et al. (2004) have been shown to suffer from a similar limitation.
The estimates of Brinchmann et al. (2004) and Gilbank et al. (2010) for the total SFRs and local density of star formation remain largely correct despite uncertainties in aperture-loss corrections for individual galaxies. Measurements using other techniques that do not require aperture corrections have already confirmed this (e.g. radio: Hopkins et al. 2003; ultraviolet: Salim et al. 2007; summary in Hopkins \& Beacom 2006). Even simple corrections deliver reasonable statistical results because the range in aperture loss is typically smaller than the range in SFRs seen in galaxies.

Despite their general correctness, aperture-loss corrections of Brinchmann et al. (2004) do systematically bias estimates of star formation. We have shown a systematic underestimate of SFRs in individual galaxies for galaxies with higher $\left(>1 \mathrm{M}_{\odot} \mathrm{yr}^{-1}\right)$ intrinsic rates of star formation. This systematic has also been seen by Chang et al. (2015) and Richards et al. (2016a) and is consistent with Gerssen et al. (2012) (who have selected only more highly starforming galaxies with $\mathrm{H} \alpha$ equivalent width greater than $20 \AA$ ). This systematic effect could lead to an underestimate of the local density of star formation by a factor of 3 - large but not grossly inconsistent with the scatter in existing estimates from different techniques. However, analysis using star formation indicators in the ultraviolet, far-infrared and radio surveys does not show this systematic bias (e.g. Hopkins et al. 2003; Salim et al. 2007). The disagreement between analysis based on optical IFS and other wavelengths is puzzling and deserves further investigation.

We recommend that all aperture-loss corrections be treated with caution, particularly for more extreme systems. The methods of Brinchmann et al. (2004) and Gilbank et al. (2010) are more representative of SFRs for individual galaxies than if aperture losses were ignored, but at the expense of introducing systematic effects in samples. Aperture-loss corrections for SFRs are far less critical for finding trends because the correction is typically smaller than the range of the SFR, which is not true for many other quantities, e.g. metallicity.

Our sample is still fairly small, and further work in this area with larger surveys using IFS may improve corrections for aperture losses. Of particular interest would be to test further for possible correlations of aperture correction with dust content and galaxy stellar mass. Also unknown, and a weakness of our analysis here, is how dust extinction may change in the outer parts of a galaxy. Current and future integral-field spectroscopic surveys such as the SAMI Galaxy Survey (Bryant et al. 2015, initial results on this topic in Richards et al. 2016a) and MaNGA (Bundy et al. 2015), with samples of 3400 and 10000 respectively, should answer these questions more thoroughly. Larger samples should also provide insight into how to improve aperture-loss corrections a priori, permitting new science with existing fibre-based surveys.

\section{ACKNOWLEDGEMENTS}

KG acknowledges support from the Australian Research Council (ARC) Discovery Program (DP) grant DP130101460. DGG acknowledges support from the National Research Foundation of South Africa.

AWG wrote the paper and did much of the new analysis. KG provided extensive scientific advice, and helped with the direction and content of the paper. DGG provided data necessary for the recomputation of the local SFR density, and helped with the correct use of these data and the Gilbank et al. (2010) results. DGG also provided the data for the analysis of the Appendix A. PJM, ID, RGA and RS assisted with the data collection and measurements on which this paper is based. All except PJM read and commented on 
the final draft - PJM unfortunately passed away before this paper was completed.

\section{REFERENCES}

Boissier S., Boselli A., Buat V., Donas J., Milliard B., 2004, A\&A, 424, 465 Brinchmann J., Charlot S., White S. D. M., Tremonti C., Kauffmann G., Heckman T., Brinkmann J., 2004, MNRAS, 351, 1151

Bryant J. J. et al., 2015, MNRAS, 447, 2857

Bundy K. et al., 2015, ApJ, 798, 7

Calzetti D., Kinney A. L., Storchi-Bergmann T., 1996, ApJ, 458, 132

Calzetti D., Armus L., Bohlin R. C., Kinney A. L., Koornneef J., StorchiBergmann T., 2000, ApJ, 533, 682

Chabrier G., 2003, PASP, 115, 763

Chang Y.-Y., van der Wel A., da Cunha E., Rix H.-W., 2015, ApJS, 219, 8

Charlot S., Kauffmann G., Longhetti M., Tresse L., White S. D. M., Maddox S. J., Fall S. M., 2002, MNRAS, 330, 876

Gerssen J., Wilman D. J., Christensen L., 2012, MNRAS, 420, 197

Gilbank D. G., Baldry I. K., Balogh M. L., Glazebrook K., Bower R. G., 2010, MNRAS, 405, 2594

Green A. W. et al., 2014, MNRAS, 437, 1070

Hopkins A. M., 2004, ApJ, 615, 209

Hopkins A. M., Beacom J. F., 2006, ApJ, 651, 142

Hopkins A. M. et al., 2003, ApJ, 599, 971

Hoversten E. A., Glazebrook K., 2008, ApJ, 675, 163

Kauffmann G. et al., 2003, MNRAS, 341, 33

Kaufman M., Bash F. N., Kennicutt R. C., Jr, Hodge P. W., 1987, ApJ, 319, 61

Kennicutt R. C., Jr, 1998, ARA\&A, 36, 189
Kennicutt R. C., Jr, Keel W. C., Blaha C. A., 1989, AJ, 97, 1022

Kewley L. J., Jansen R. A., Geller M. J., 2005, PASP, 117, 227

Kroupa P., 2001, MNRAS, 322, 231

Peacock J. A., 1983, MNRAS, 202, 615

Pei Y. C., 1992, ApJ, 395, 130

Richards S. N. et al., 2016a, MNRAS, 455, 2826

Richards S. N. et al., 2016b, MNRAS, 458, 1300

Robotham A. S. G., Obreschkow D., 2015, Publ. Astron. Soc. Aust., 32, e033

Sadler E. M., 2002, MNRAS, 329, 227

Salim S. et al., 2007, ApJS, 173, 267

Salpeter E. E., 1955, ApJ, 121, 161

Sullivan M., Treyer M. A., Ellis R. S., Bridges T. J., Milliard B., Donas J., 2000, MNRAS, 312, 442

Tremonti C. A. et al., 2004, ApJ, 613, 898

Wild V., Charlot S., Brinchmann J., Heckman T., Vince O., Pacifici C., Chevallard J., 2011, MNRAS, 417, 1760

York D. G. et al., 2000, AJ, 120, 1579

\section{APPENDIX A: CHANGES IN APERTURE CORRECTIONS IN DR7}

This paper has used the Brinchmann et al. (2004) SFRs and aperture corrections calculated for the Data Release 4 (DR4) of the SDSS. This appendix shows how changes introduced in the rates and corrections calculated for the Data Release 7 (DR7) of SDSS affect our results.

A test of SDSS aperture corrections with IFS
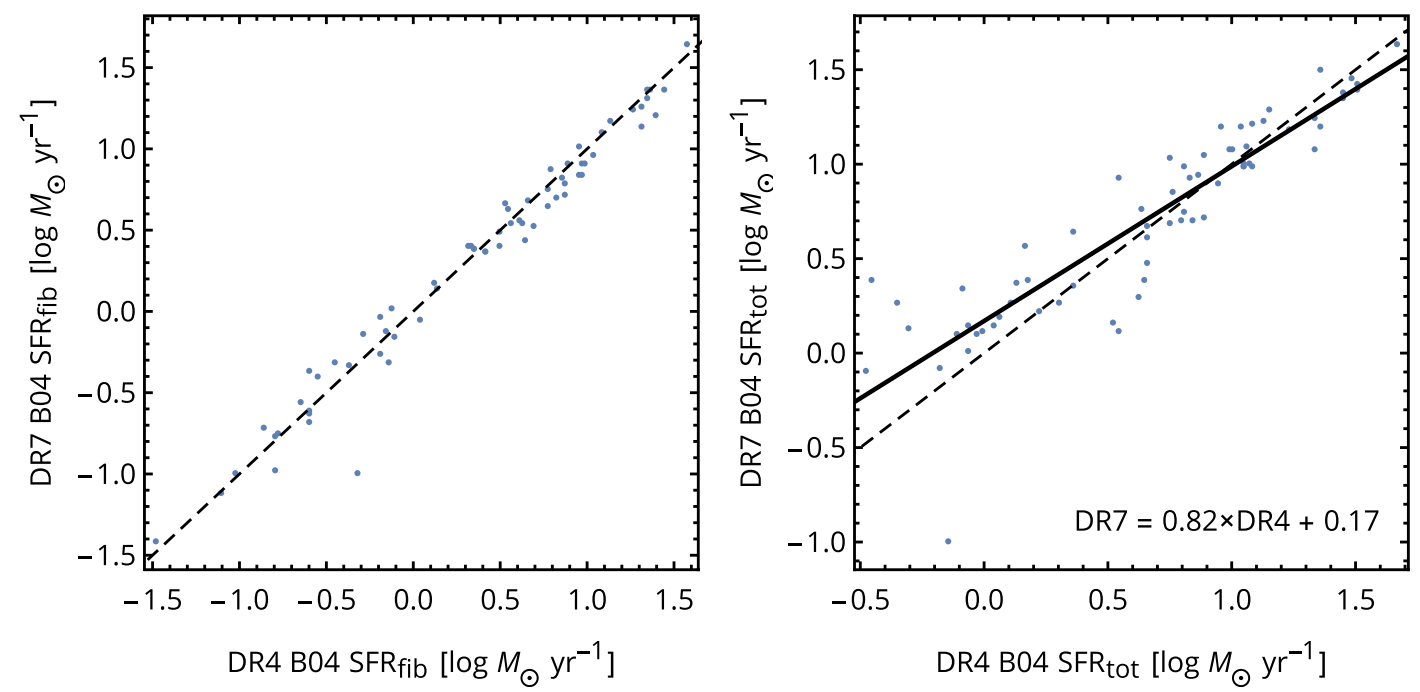

Figure A1. Comparison of fibre aperture (left) and total (right) SFRs using the Brinchmann et al. (2004) methods as applied to DR4 and DR7. The version of the method used for DR7 differs slightly from that of DR4. The one-to-one relation is shown as a dashed line in each panel. The fibre-aperture SFRs agree very well, but the aperture-corrected total SFRs show a slight systematic for our galaxies as shown by the best-fitting solid line in the right-hand panel. 
For the release of the DR7 Value Added Catalog, two changes were introduced ${ }^{8}$ that could affect the calculation of the aperture correction. The first change addresses a modification to the spectrophotometry calibration in the underlying SDSS data - the MPAJHU group renormalized the spectra. This change should only have a minor impact on the aperture correction, if any at all. The second change addresses issues with some galaxy classes raised by Salim et al. (2007). They noted a problem in the aperture corrections to non-star-forming galaxies, which were traced to an issue with photometry. This issue does not affect galaxies classified as star forming. To address this issue, the MPA-JHU group changed the calculation of the photometry used in determining the aperture corrections for all galaxies. As we will see these small changes only strengthen the conclusions of this paper.

Fig. A1 shows the comparison between DR4 and DR7 for SFRs computed according to Brinchmann et al. (2004). The DR7 rates include the changes mentioned above. A slight systematic difference is apparent in the total SFRs between DR4 and DR7 - DR7 rates are higher for galaxies with lower SFRs.

The difference between DR7 and DR4 SFRs affects the results of this paper in several ways. DR7 SFRs are further underestimated for highly star-forming galaxies. Fig. A2 reproduces the left-hand panel of Fig. 3 updated for DR7. The slope of the best-fitting line drops from 0.83 to 0.75 .

\footnotetext{
${ }^{8}$ Described online at http://wwwmpa.mpa-garching.mpg.de/SDSS/DR7/ index.html.
}

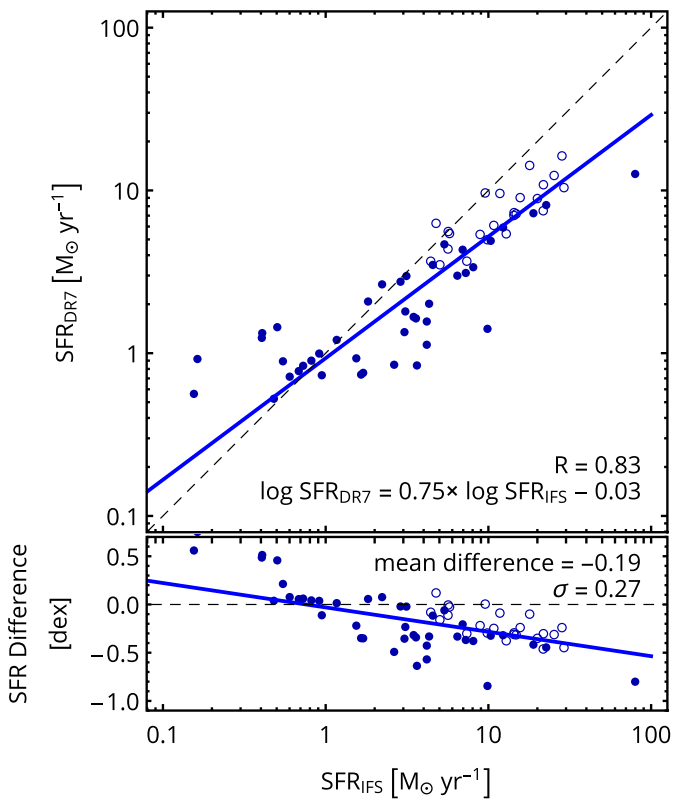

Figure A2. The left-hand panel of Fig. 3 reproduced using the DR7 version of the MPA-JHU Value Added Catalog instead of the DR4 version.

This appendix has shown that the changes introduced into the aperture-correction method of Brinchmann et al. (2004) for DR7 further strengthen the findings of this paper. Therefore, our primary conclusion - aperture-loss corrections should be treated with caution - remains valid.

This paper has been typeset from a $\mathrm{T}_{\mathrm{E}} \mathrm{X} / \mathrm{L} \mathrm{T}_{\mathrm{E}} \mathrm{X}$ file prepared by the author. 